\title{
Dopamine receptor heteromers: biasing antipsychotics
}

\author{
Kristoffer Sahlholm*,1,2, Marta Valle-León ${ }^{1,3}$, Víctor Fernández-Dueñas ${ }^{1,3}$ \& Francisco \\ Ciruela**,1,3 \\ ${ }^{1}$ Unitat de Farmacologia, Departament Patologia i Terapèutica Experimental, Facultat de Medicina i Ciències de la Salut, \\ IDIBELL-Universitat de Barcelona, L'Hospitalet de Llobregat, Spain \\ ${ }^{2}$ Department of Neuroscience, Karolinska Institutet, Stockholm, Sweden \\ ${ }^{3}$ Institut de Neurociències, Universitat de Barcelona, Barcelona, Spain \\ * Author for correspondence: kristoffer@sahlholm@ki.se \\ **Author for correspondence: fciruela@ub.edu
}

\begin{abstract}
' the development of purely allosteric ligands, which could bias dopamine signaling without competing for binding to the orthosteric site, might be a preferential strategy over the recently developed orthosteric ligands"”
\end{abstract}

First draft submitted: 17 July 2018; Accepted for publication: 24 October 2018; Published online: 6 December 2018

Keywords: antipsychotic $\bullet$ dopamine $D_{2}$ receptor

The term antipsychotic drug (APD), also called a neuroleptic, refers to medications currently prescribed for the treatment of neuropsychiatric diseases that display psychotic features (e.g., schizophrenia) [1]. Classification of APDs is mainly based on the profile of their adverse effects; in particular, liability to produce extrapyramidal symptoms (EPS). Accordingly, typical (or first generation) and atypical (or second generation) drugs have been differentiated. Typical APDs are effective at controlling the so-called positive symptoms of schizophrenia; hallucinations and delusions, but they are more likely to produce undesired motor effects than atypical APDs [2]. However, this kind of classification has been questioned and, in fact, each APD may be considered as having a unique set of properties [3]. Meanwhile, it is important to note that a substantial number of patients do not respond adequately to existing APDs, and in particular, the negative symptoms of schizophrenia, which include cognitive deficits, social withdrawal, anhedonia and avolition, are hardly ameliorated by current treatments. Thus, there exists a clinical need for the development of mechanistically novel and more efficacious APDs.

\section{The dopaminergic hypothesis of schizophrenia}

It is well accepted that dysregulation of dopaminergic neurotransmission is central to most psychotic processes, including schizophrenia. The dopaminergic hypothesis concerning schizophrenia states that hyperactivity in the mesolimbic dopamine pathway concurs with hypofunction in the mesocortical dopamine pathway [4]. Accordingly, reducing signaling via mesolimbic dopamine $\mathrm{D}_{2}$ receptors $\left(\mathrm{D}_{2} \mathrm{Rs}\right)$ is probably the main mechanism through which antipsychotic therapeutics function. Indeed, $A P D s$ are either antagonists or partial agonists for $\mathrm{D}_{2} \mathrm{Rs}$. Importantly, the level of antipsychotic occupancy of striatal $\mathrm{D}_{2} \mathrm{Rs}$ is related to the appearance of adverse motor effects such as EPS; neuroimaging studies have revealed that a $\mathrm{D}_{2} \mathrm{R}$ occupancy of above $80 \%$ is associated with the appearance of EPS [5]. Interestingly, while typical APDs such as haloperidol may result in this degree of occupancy at therapeutic doses, atypical ones such as clozapine achieve antipsychotic efficacy at lower occupancy levels, which may explain why they are less likely to produce EPS [5].

\section{$D_{2} R$-mediated $\beta$-arrestin2 signaling \& antipsychotic-like activity}

$\mathrm{D}_{2} \mathrm{Rs}$ are $\mathrm{G}$-protein-coupled receptors that signal via Gi/o and $\beta$-arrestin2 proteins [6]. It has been suggested that the therapeutic benefits of APDs may be specifically related to the inhibition of $D_{2} R$-mediated $\beta$-arrestin activation [7]; a mechanistic feature shared by both typical and atypical APDs. Furthermore, it was hypothesized that induction of EPS may result from an excessive reduction of G-protein-dependent signaling downstream of 
$\mathrm{D}_{2} \mathrm{R}$. Interestingly, expression of $\beta$-arrestin 2 and also of $G$ protein receptor kinase-2 (GRK2), which phosphorylates $\mathrm{D}_{2} \mathrm{R}$ and enhances $\beta$-arrestin2 recruitment has been shown to be lower in striatum than in cortex [8]. These data are consistent with functional magnetic resonance imaging studies in mice, showing that dopaminergic stimulation elicits an arrestin-dependent response in cortex, but not in striatum [9]. These regional differences may support the use of weak partial D2R agonists, which preferentially signal via the $\beta$-arrestin2, as opposed to the G protein, pathway such as $\beta$-arrestin2-biased agonists to induce D2R-mediated $\beta$-arrestin2 signaling in cortex selectively, counteracting the hypodopaminergia which is believed to underlie negative symptoms, while antagonizing elevated dopamine signaling in the striatum thought to provoke positive symptoms [10]. In addition, it would be beneficial not to interfere with $G$ protein signaling since this could potentially avoid EPS.

Recent studies [10,11] reported antipsychotic-like activity (i.e., reduction of PCP- and amphetamine-induced hyperlocomotion) of $\beta$-arrestin2-biased aripiprazole analogs in mice. These $\mathrm{D}_{2} \mathrm{R}$ ligands showed a low propensity to induce catalepsy (used as an indicator of EPS liability). In addition, the antipsychotic-like activity was abolished in $\beta$-arrestin 2 deficient animals, supporting the notion that $\beta$-arrestin2 plays a key role in the effects elicited by the aripiprazole derivatives. In the same work [10], one of the biased ligands, UNC9994, was reportedly unable to interact as either an agonist or an antagonist with G-protein-dependent signaling downstream of $\mathrm{D}_{2} \mathrm{Rs}$. This kind of behavior suggests that UNC9994 is unable to compete with dopamine at receptors coupled to G protein. However, such a mechanism appears unlikely, since, based on its aripiprazole-like structure, this ligand is expected to interact with the orthosteric binding site of $\mathrm{D}_{2} \mathrm{R}$. Indeed, recent data indicates that UNC9994 is a weak partial agonist at $G$ protein-dependent potassium channel activation downstream of $D_{2} R s$ and, as expected for a weak partial agonist, antagonizes the response to a near-maximally effective concentration of dopamine [12].

\section{Impact of $D_{2} R$ heteromerization in antipsychotic drug efficacy}

Apart from the differential expression of $\beta$-arrestin2 and GRK2 in cortical and striatal brain areas, the biased effects of $\mathrm{D}_{2} \mathrm{R}$ ligands may depend on other interacting proteins, such as $\mathrm{G}$-protein-coupled receptors. $\mathrm{D}_{2} \mathrm{R}$ functioning in the striatum has been reported to be finely modulated by adenosine $A_{2 A}$ receptors $\left(A_{2 A} R s\right)$. Indeed, the existence of direct receptor-receptor interactions (in other words, heteromerization) between $\mathrm{D}_{2}$ Rs and $\mathrm{A}_{2 \mathrm{~A}} \mathrm{Rs}$, in which the protomers display reciprocal negative allosteric modulation, has been well established [13]. Conversely, the expression of $A_{2 A}$ Rs in cortex is very low compared to that observed in striatum [14]; accordingly, it seems unlikely that $\mathrm{D}_{2}$ Rs participate in $\mathrm{D}_{2} \mathrm{R} / \mathrm{A}_{2 \mathrm{~A}} \mathrm{R}$ heteromers in this brain region, in spite of what was recently demonstrated [15]. Within this context, the antipsychotic-like efficacy of UNC9994 was recently assessed in $\mathrm{A}_{2 \mathrm{~A}} \mathrm{R}$-deficient $\left(\mathrm{A}_{2 \mathrm{~A}} \mathrm{R}^{-/-}\right)$ mice, in order to study its possible dependence on $A_{2 A} R$ expression [16]. Interestingly, UNC9994 exerted robust antipsychotic-like effects in wild-type, but not in $\mathrm{A}_{2 \mathrm{~A}} \mathrm{R}^{-/-}$, animals; thus, the compound failed to abolish PCPinduced hyperlocomotion in $\mathrm{A}_{2 \mathrm{~A}} \mathrm{R}^{-/-}$mice, and reduced amphetamine-induced hyperlocomotion to a lesser extent in these animals, than in wild-type mice [16]. Taken altogether, and since UNC9994 mainly activates the $\beta$-arrestin2 signaling pathway, it was concluded that $A_{2 A} R$ expression might determine the biased nature of the ligand, by driving the formation of a striatal $D_{2} R / A_{2 A} R / \beta$-arrestin2 complex. However, we cannot rule out an effect on $G$ protein signaling, especially since $\mathrm{A}_{2 \mathrm{~A}} \mathrm{R}^{-/-}$mice are less sensitive to the suppressing effects of antipsychotics on basal locomotion [17].

In summary, biased antipsychotic ligands represent an interesting avenue for future research. However, the development of purely allosteric ligands, which could bias dopamine signaling without competing for binding to the orthosteric site, might be a preferential strategy over the recently developed orthosteric ligands. Indeed, a series of negative allosteric $\mathrm{D}_{2} \mathrm{R}$ modulators, which do not interact with the orthosteric site, but modulate dopamine efficacy, has recently been reported [18]. Such ligands may serve as lead compounds for the development of biased allosteric modulators, which could potentially evolve into more efficacious APDs. In addition, understanding the impact of $D_{2} R / A_{2 A} R$ heteromer formation on $D_{2} R$ downstream signaling will certainly contribute to the development of such novel APDs.

\section{Financial \& competing interests disclosure}

The authors have no relevant affiliations or financial involvement with any organization or entity with a financial interest in or financial conflict with the subject matter of or materials discussed in this manuscript. This includes employment, consultancies, honoraria, stock ownership or options, expert testimony, grants or patents received or pending, and royalties.

No writing assistance was utilized in the production of this manuscript. 


\section{References}

1. Miyamoto S, Miyake N, Jarskog LF, Fleischhacker WW, Lieberman JA. Pharmacological treatment of schizophrenia: a critical review of the pharmacology and clinical effects of current and future therapeutic agents. Mol. Psychiatry 17(12), 1206-1227 (2012).

2. Meltzer HY. Update on typical and atypical antipsychotic drugs. Annu. Rev. Med. 64(1), 393-406 (2013).

3. Gründer G, Hippius H, Carlsson A. The 'atypicality' of antipsychotics: a concept re-examined and re-defined. Nat. Rev. Drug Discov. 8(3), 197-202 (2009).

4. Heinz A, Schlagenhauf F. Dopaminergic dysfunction in schizophrenia: salience attribution revisited. Schizophr. Bull. 36(3), 472-85 (2010).

5. Uchida H, Takeuchi H, Graff-Guerrero A, Suzuki T, Watanabe K, Mamo DC. Predicting dopamine D2 receptor occupancy from plasma levels of antipsychotic drugs. J. Clin. Psychopharmacol. 31(3), 318-325 (2011).

6. Quan W, Kim J-H, Albert PR, Choi H, Kim K-M. Roles of G protein and beta-arrestin in dopamine D2 receptor-mediated ERK activation. Biochem. Biophys. Res. Commun. 377(2), 705-709 (2008).

7. Masri B, Salahpour A, Didriksen M et al. Antagonism of dopamine D2 receptor/ -arrestin 2 interaction is a common property of clinically effective antipsychotics. Proc. Natl Acad. Sci. USA 105(36), 13656-13661 (2008).

8. Urs NM, Peterson SM, Caron MG. New concepts in dopamine D2 receptor biased signaling and implications for schizophrenia therapy. Biol. Psychiatry 81(1), 78-85 (2017).

9. Sahlholm K, Ielacqua GD, Xu J et al. The role of beta-arrestin2 in shaping fMRI BOLD responses to dopaminergic stimulation. Psychopharmacolgy 234(13), 2019-2030 (2017).

10. Urs NM, Gee SM, Pack TF et al. Distinct cortical and striatal actions of a $\beta$-arrestin-biased dopamine D2 receptor ligand reveal unique antipsychotic-like properties. Proc. Natl Acad. Sci. USA 113(50), E8178-E8186 (2016).

11. Allen JA, Yost JM, Setola V et al. Discovery of $\beta$-arrestin-biased dopamine D2 ligands for probing signal transduction pathways essential for antipsychotic efficacy. Proc. Natl Acad. Sci. USA 108(45), 18488-18493 (2011).

12. Ågren R, Århem P, Nilsson J, Sahlholm K. The beta-arrestin-biased dopamine D2 receptor ligand, UNC9994, is a partial agonist at G-protein-mediated potassium channel activation. Int. J. Neuropsychopharmacol. 21(12), 1102-1108 (2018).

13. Ferré S, Ciruela F, Woods AS, Lluis C, Franco R. Functional relevance of neurotransmitter receptor heteromers in the central nervous system. Trends Neurosci. 30(9), 440-446 (2007).

14. Fredholm BB, IJzerman AP, Jacobson KA, Linden J, Muller CE. International Union of Basic and Clinical Pharmacology. LXXXI. Nomenclature and classification of adenosine receptors - an update. Pharmacol. Rev. 63(1), 1-34 (2011).

15. Fernández-Dueñas V, Taura JJ, Cottet $\mathrm{M}$ et al. Untangling dopamine-adenosine receptor-receptor assembly in experimental parkinsonism in rats. Dis. Model. Mech. 8(1), 57-63 (2015).

16. Sahlholm K, Gómez-Soler M, Valle-León M et al. Antipsychotic-like efficacy of dopamine D2 receptor-biased ligands is dependent on adenosine a2a receptor expression. Mol. Neurobiol. 55(6), 4952-4958 (2018).

17. Pardo M, López-Cruz L, Valverde $\mathrm{O}$ et al. Effect of subtype-selective adenosine receptor antagonists on basal or haloperidol-regulated striatal function: studies of exploratory locomotion and c-Fos immunoreactivity in outbred and A2AR KO mice. Beh. Brain Res. 247, 217-226 (2013).

18. Fyfe TJ, Zarzycka B, Lim HD et al. A thieno[2,3- d]pyrimidine scaffold is a novel negative allosteric modulator of the dopamine D2 receptor. J. Med. Chem., acs.jmedchem.7b01565 (2018) (Epub ahead of print). 
